\title{
Lactic Acid Bacteria as Probiotics: Characteristics, Selection Criteria and Role in Immunomodulation of Human GI Muccosal Barrier
}

\author{
Daoud Harzallah and Hani Belhadj \\ Additional information is available at the end of the chapter
}

\section{Introduction}

As it was reported by Chow (2002), the notion that food could serve as medicine was first conceived thousands of years ago by the Greek philosopher and father of medicine, Hippocrates, who once wrote: 'Let food be thy medicine, and let medicine be thy food'. However, during recent times, the concept of food having medicinal value has been reborn as 'functional foods'. The list of health benefits accredited to functional food continues to increase, and the gut is an obvious target for the development of functional foods, because it acts as an interface between the diet and all other body functions. One of the most promising areas for the development of functional food components lies in the use of probiotics and prebiotics which scientific researches have demonstrated therapeutic evidence. Nowadays, consumers are aware of the link among lifestyle, diet and good health, which explains the emerging demand for products that are able to enhance health beyond providing basic nutrition. Besides the nutritional valaes, ingestion of lactic acid bacteria (LAB) and their fermented foods has been suggested to confer a range of health benefits including immune system modulation, increased resistance to malignancy, and infectious illness (Soccol, et al., 2010). LAB were first isolated from milk. They can be found in fermented products as meat, milk products, vegetables, beverages and bakery products. LAB occur naturally in soil, water, manure, sewage, silage and plants. They are part of the microbiota on mucous membranes, such as the intestines, mouth, skin, urinary and genital organs of both humans and animals, and may have a beneficial influence on these ecosystems. LAB that grow as the adventitious microflora of foods or that are added to foods as cultures are generally considered to be harmless or even an advantage for human health. Since their discovery, LAB has been gained mush 
interest in various applications, as starter cultures in food and feed fermentations, pharmaceuticals, probiotics and as biological control agents. In food industry, LAB are widely used as starters to achieve favorable changes in texture, aroma, flavor and acidity (Leory and De Vuyst, 2004). However, there has been an important interest in using bacteriocin and/or other inhibitory substance producing LAB for non-fermentative biopreservation applications. Du to their antimicrobial and antioxidant activities some LAB strains are used in food biopreservation. However, LAB are generally regarded as safe (GRAS) to the consumer and during storage, they naturally dominate the microflora of many foods (Osmanağaoğlu and Beyatli, 1999; Parada et al., 2007). Many of the indications for probiotic activity have been obtained from effects observed in various clinical situations. Even, there are few strains that have officially gained the status of pharmaceutical preparation; each of these effects is gradually being supported by a number of clinical studies or human intervention trials, performed in a way that resembles the traditional pharmacological approach (placebo-controlled, double blind, randomized trials) and the strains used in these studies belong to different microbial species, but are mostly lactic acid bacteria (Mercenier et al, 2003).

\section{LAB as probiotic agents}

\subsection{Overview of probiotics}

The most tried and tested manner in which the gut microbiota composition may be influenced is through the use of live microbial dietary additions, as probiotics. In fact, the concept dates back as far as prebiblical ages (Rastall et al., 2000). The first records of ingestion of live bacteria by humans are over 2,000 years old. However, at the beginning of this century probiotics were first put onto a scientific basis by the work of Metchnikoff (1908). He hypothesised that the normal gut microflora could exert adverse effects on the host and that consumption of 'soured milks' reversed this effect. The word "probiotics" was initially used as an anonym of the word "antibiotic". It is derived from Greek words pro and biotos and translated as "for life". The origin of the first use can be traced back to Kollath (1953), who used it to describe the restoration of the health of malnourished patients by different organic and inorganic supplements. Later, Vergin (1954) proposed that the microbial imbalance in the body caused by antibiotic treatment could have been restored by a probiotic rich diet; a suggestion cited by many as the first reference to probiotics as they are defined nowadays. Similarly, Kolb recognized detrimental effects of antibiotic therapy and proposed the prevention by probiotics (Vasiljevic and Shah, 2008) Later on, Lilly and Stillwell (1965) defined probiotics as 'microorganisms promoting the growth of other microorganisms'. Following recommendations of a FAO/WHO (2002) working group on the evaluation of probiotics in food, probiotics, are live microorganisms that, when administered in adequate amounts, confer a health benefit on the host (Sanders, 2008; Schrezenmeir and De Vrese, 2001). The idea of health-promoting effects of LAB is by no means new, as Metchnikoff proposed that lactobacilli may fight against intestinal putrefaction and contribute to long life. Such microorganisms may not necessarily be constant inhabitants of the gut, but they should have a "beneficial effect on the general and health status of man and animal". 
(Holzapfel et al., 2001; Belhadj et al., 2010). Other definitions advanced through the years have been restrictive by specification of mechanisms, site of action, delivery format, method, or host. Probiotics have been shown to exert a wide range of effects. The mechanism of action of probiotics (e.g, having an impact on the intestinal microbiota or enhancing immune function) was dropped from the definition to encompass health effects due to novel mechanisms and to allow application of the term before the mechanism is confirmed. Physiologic benefits have been attributed to dead microorganisms. Furthermore, certain mechanisms of action (such as delivery of certain enzymes to the intestine) may not require live cells. However, regardless of functionality, dead microbes are not probiotics (Sanders, 2008). In relation to food, probiotics are considered as "viable preparations in foods or dietary supplements to improve the health of humans and animals". According to these definitions, an impressive number of microbial species are considered as probiotics. (Holzapfel et al., 2001). For gastrointestinal ecosysteme, however, the most important microbial species that are used as probiotics are lactic acid bacteria (LAB) (Table 1).

\subsection{Selection of probiotics}

Many in vitro tests are performed when screening for potential probiotic strains. The first step in the selection of a probiotic LAB strain is the determination of its taxonomic classification, which may give an indication of the origin, habitat and physiology of the strain. All these characteristics have important consequences on the selection of the novel strains (Morelli, 2007). An FAO/WHO (2002) expert panel suggested that the specificity of probiotic action is more important than the source of microorganism. This conclusion was brought forward due to uncertainty of the origin of the human intestinal microflora since the infants are borne with virtually sterile intestine. However, the panel also underlined a need for improvement of in vitro tests to predict the performance of probiotics in humans. While many probiotics meet criteria such as acid and bile resistance and survival during gastrointestinal transit, an ideal probiotic strain remains to be identified for any given indication. Furthermore, it seems unlikely that a single probiotic will be equally suited to all indications; selection of strains for disease-specific indications will be required (Shanahan, 2003).

The initial screening and selection of probiotics includes testing of the following important criteria: phenotype and genotype stability, including plasmid stability; carbohydrate and protein utilization patterns; acid and bile tolerance and survival and growth; intestinal epithelial adhesion properties; production of antimicrobial substances; antibiotic resistance patterns; ability to inhibit known pathogens, spoilage organisms, or both; and immunogenicity. The ability to adhere to the intestinal mucosa is one of the more important selection criteria for probiotics because adhesion to the intestinal mucosa is considered to be a prerequisite for colonization (Tuomola et al., 2001). The table below (Table 2) indicates key creteria for sellecting probiotic candidat for commercial application, and Figure $\mathbf{1}$ presents major and cardinal steps for sellecting probiotic candidats.

It is of high importance that the probiotic strain can survive the location where it is presumed to be active. For a longer and perhaps higher activity, it is necessary that the strain 


\begin{tabular}{|c|c|c|}
\hline Probiotic & $\begin{array}{l}\text { Human disease in which } \\
\text { benefit is shown }\end{array}$ & $\begin{array}{l}\text { Animal model in which benefit is } \\
\text { shown }\end{array}$ \\
\hline \multicolumn{3}{|l|}{ Yeast } \\
\hline Saccharomyces boulardii & Clostridium difficile infection & Citrobacter rodentium-induced colitis \\
\hline \multicolumn{3}{|l|}{ Gram-negative bacteria } \\
\hline Escherichia coli Nissle 1917 & NA & DSS-induced colitis \\
\hline \multicolumn{3}{|l|}{ Gram-positive bacteria } \\
\hline Bifidobacteria bifidum & NA & $\begin{array}{l}\text { Rat model of necrotizing } \\
\text { enterocolitis }\end{array}$ \\
\hline Bifidobacteria infantis & IBS29 & NA \\
\hline $\begin{array}{l}\text { Lactobacillus rhamnosus GG } \\
\text { (used with lactoferrin) }\end{array}$ & $\begin{array}{l}\text { Sepsis in very low birth } \\
\text { weight infants }\end{array}$ & NA \\
\hline $\begin{array}{l}\text { Lactococcus lactis (engineered to } \\
\text { produce IL-10 or trefoil factors) }\end{array}$ & Crohn's disease & $\begin{array}{l}\text { DSS-induced colitis and IL- } 10^{-/} \text {mice } \\
\text { (spontaneous IBD) }\end{array}$ \\
\hline Lactobacillus plantarum $299 \mathrm{v}$ & Antibiotic-associated diarrhea & IL-10-/ mice (spontaneous IBD) \\
\hline Lactobacillus acidophilus & NA & $\begin{array}{l}\text { Visceral hyperalgesia } 40 \text { and } \\
\text { C. rodentium-induced colitis }\end{array}$ \\
\hline Lactobacillus rhamnosus & $\begin{array}{l}\text { Pediatric antibiotic-associated } \\
\text { diarrhea }\end{array}$ & - \\
\hline Lactobacillus casei & NA & DNBS-induced colitis \\
\hline Bacillus polyfermenticus & NA & $\begin{array}{l}\text { DSS-induced colitis and TNBS- } \\
\text { induced colitis }\end{array}$ \\
\hline \multicolumn{3}{|l|}{ Combination regimens } \\
\hline $\begin{array}{l}\text { Lactobacillus rhamnosus GG combined with } \\
\text { Bifidobacterium lactis }\end{array}$ & Bacterial infections & NA \\
\hline $\begin{array}{l}\text { Lactobacillus rhamnosus combined with } \\
\text { Lactobacillus helveticus }\end{array}$ & NA & $\begin{array}{l}\text { C. rodentium-induced colitis, chronic } \\
\text { stress, and early life stress }\end{array}$ \\
\hline $\begin{array}{l}\text { VSL\#3 (Lactobacillus casei, Lactobacillus } \\
\text { plantarum, Lactobacillus acidophilus, } \\
\text { Lactobacillus bulgaricus, Bifidobacterium longum, } \\
\text { Bifidobacterium breve, Bifidocacterium infantis and } \\
\text { Streptococcus thermophilus) }\end{array}$ & $\begin{array}{l}\text { Pouchitis and pediatric } \\
\text { ulcerative colitis }\end{array}$ & $\begin{array}{l}\text { DSS-induced colitis, IL-10-/ mice } \\
\text { (spontaneous IBD; DNA only), } \\
\text { and SAMP mouse model of } \\
\text { spontaneous IBD }\end{array}$ \\
\hline
\end{tabular}

Abbreviations: DNBS, dinitrobenzene sulfonic acid; DSS, dextran sodium sulfate; IL-10, interleukin 10; NA, not available; TNBS, trinitrobenzene sulfonic acid.

Table 1. Selected organisms that are used as probiotic agents (Gareau et al., 2010).

can proliferate and colonise at this specific location. Probably only host-specific microbial strains are able to compete with the indigenous microflora and to colonise the niches. Besides, the probiotic strain must be tolerated by the immune system and not provoke the formation of antibodies against the probiotic strain. So, the host must be immunotolerant to the probiotic. On the other hand, the probiotic strain can act as an adjuvant 


\begin{tabular}{ll}
\hline General & Property \\
Safety criteria & Origin \\
& Pathogenicity and infectivity \\
& Virulence factors - toxicity, metabolic activity and intrinsic properties, i.e., \\
antibiotic resistance & \\
& Genetically stable strains \\
& Desired viability during processing and storage \\
Technological criteria & Good sensory properties \\
& Phage resistance \\
& Large-scale production \\
& Tolerance to gastric acid and juices \\
& Bile tolerance \\
& Adhesion to mucosal surface \\
& Validated and documented health effects \\
Functional criteria & Immunomodulation \\
Antagonistic activity towards gastrointestinal & pathogens, i.e., Helicobacter pylori, Candida albicans \\
Cholesterol metabolism & Lactose metabolism \\
& Antimutagenic and anticarcinogenic properties \\
criteria & \\
\hline
\end{tabular}

Table 2. Key and desirable criteria for the selection of probiotics in commercial applications (Vasiljevic and Shah, 2008).

and stimulate the immune system against pathogenic microorganisms. It goes without saying that a probiotic has to be harmless to the host: there must be no local or general pathogenic, allergic or mutagenic/carcinogenic reactions provoked by the microorganism itself, its fermentation products or its cell components after decrease of the bacteria (Desai, 2008).

When probiotic strains are selected, attributes important for efficacy and technological function must be assessed and a list of characteristics required for all probiotic functions is required. Basic initial characterization of strain identity and taxonomy should be conducted, followed by evaluation with validated assays both in studies of animal models and in controlled studies in the target host. In vitro assays are frequently conducted that have not been proved to be predictive of in vivo function. Technological robustness must also be determined, such as the strain's ability to be grown to high numbers, concentrated, stabilized, and incorporated into a final product with good sensory properties, if applicable, and to be stable, both physiologically and genetically, through the end of the shelf life of the product and at the active site in the host. Assessment of stability can also be a challenge, since factors such as chain length and injury may challenge the typical assessment of colony-forming units, as well as in vivo function (Sanders, 2008). Dose levels of probiotics should be based on levels found to be efficacious in human studies. One dose level cannot 


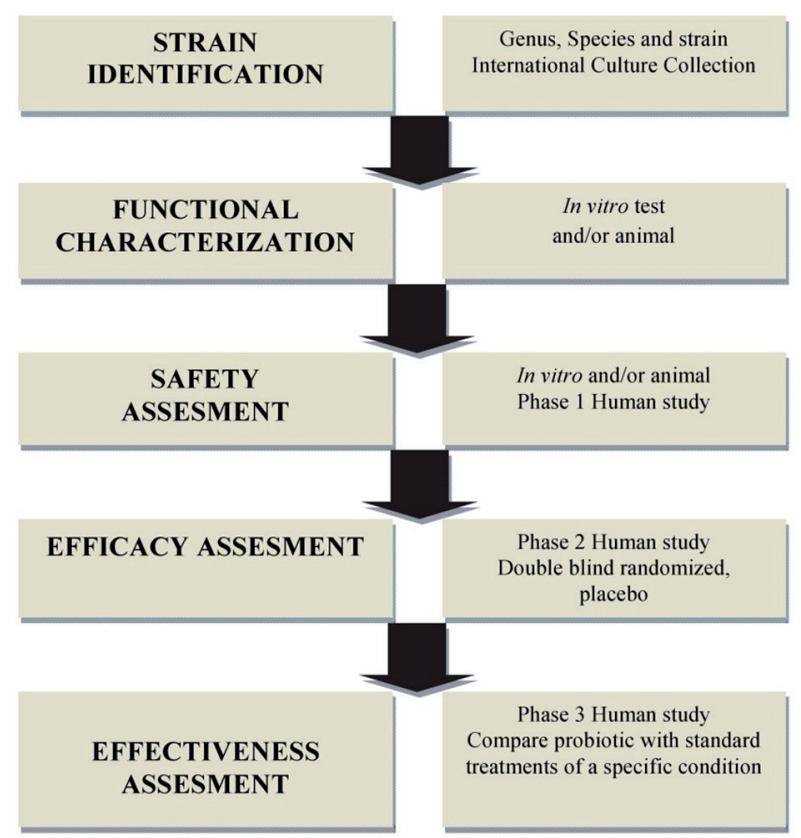

Figure 1. Scheme of the guidelines for the evaluation of probiotics for food use. (Adapted from, Collado et al., 2009).

be assumed to be effective for all strains. Furthermore, the impact of product format on probiotic function has yet to be explored in depth. The common quality-control parameter of colony-forming units per gram may not be the only parameter indicative of the efficacy of the final product. Other factors, such as probiotic growth during product manufacture, coating, preservation technology, metabolic state of the probiotic, and the presence of other functional ingredients in the final product, may play a role in the effectiveness of a product. More research is needed to understand how much influence such factors have on in vivo efficacy (Sanders, 2008).

\subsection{Potential mechanisms of action of probiotics}

A wide variety of potential beneficial health effects have been attributed to probiotics (Table 3). Claimed effects range from the alleviation of constipation to the prevention of major life-threatening diseases such as inflammatory bowel disease, cancer, and cardiovascular incidents. Some of these claims, such as the effects of probiotics on the shortening of intestinal transit time or the relief from lactose maldigestion, are considered well-established, while others, such as cancer prevention or the effect on blood cholesterol levels, need further scientific backup (Leroy et al., 2008). The mechanisms of action may vary from one probiotic strain to another and are, in most cases, probably a combination of activities, thus making the investigation of the responsible mechanisms a very difficult and complex task. In 


\author{
Health benefit \\ Cancer prevention
}

Control of irritable bowel

syndrome

Management and prevention of

atopic diseases

Management of inflammatory

bowel diseases (Crohn's disease, ulcerative colitis, pouchitis)

Prevention of heart diseases/influence on blood cholesterol levels

Prevention of urogenital tract

disorders

Prevention/alleviation of diarrhoea

caused by bacteria/viruses

Prevention/treatment of

Helicobacter pylori infections

Relief of lactose indigestion

Shortening of colonic transit time
Proposed mechanism(s)

Inhibition of the transformation of pro-carcinogens into active carcinogens, binding/inactivation of mutagenic compounds, production of anti-mutagenic compounds, suppression of growth of pro-carcinogenic bacteria, reduction of the absorption of carcinogens, enhancment of immune function, influence on bile salt concentrations

Modulation of gut microbiota, reduction of intestinal gas production

Modulation of immune response

Modulation of immune response, modulation of gut

microbiota

Assimilation of cholesterol by bacterial cells, deconjugation of bile acids by bacterial acid hydrolases,

cholesterol-binding to bacterial cell walls, reduction of hepatic cholesterol synthesis and/or redistribution of cholesterol from plasma to liver through influence of the bacterial production of short-chain fatty acids

Production of antimicrobial substances, competition for adhesion sites, competitive exclusion of pathogens

Modulation of gut microbiota, production of antimicrobial substances, competition for adhesion sites, stimulation of mucus secretion, modulation of immune response

Production of antimicrobial substances, stimulation of the mucus secretion, competition for adhesion sites, stimulation of specific and non-specific immune responses

Action of bacterial $\beta$-galactosidase(s) on lactose

Influence on peristalsis through bacterial metabolite production

Table 3. Potential and established health benefits associated with the usage of probiotics (Leroy et al., 2008).

general, three levels of action can be distinguished: probiotics can influence human health by interacting with other microorganisms present on the site of action, by strengthening mucosal barriers, and by affecting the immune system of the host (Leroy et al., 2008), and the Figure 2 shows the most important mechanisms by whiche probiotics exerce their action inside the gut. 

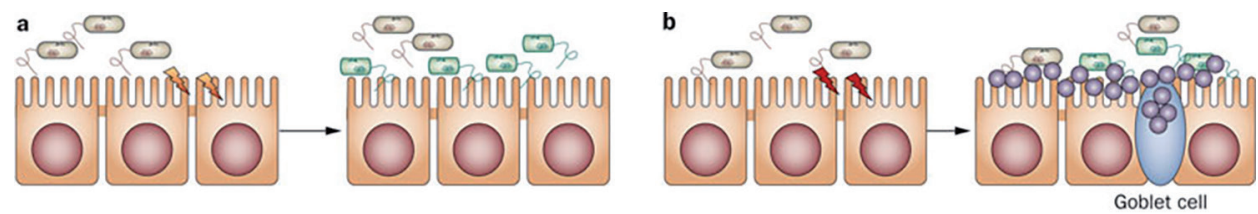

c
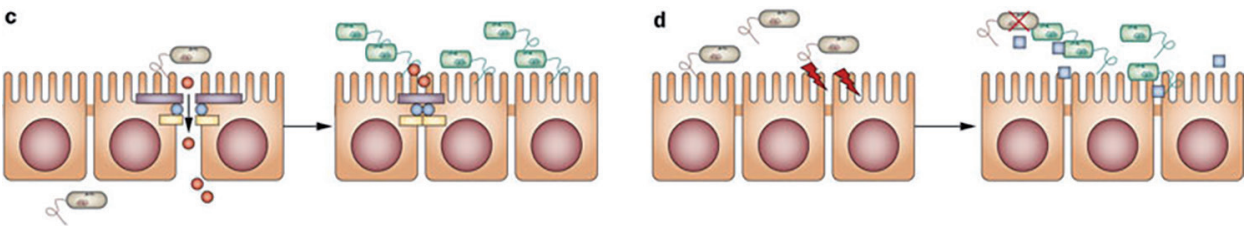

e
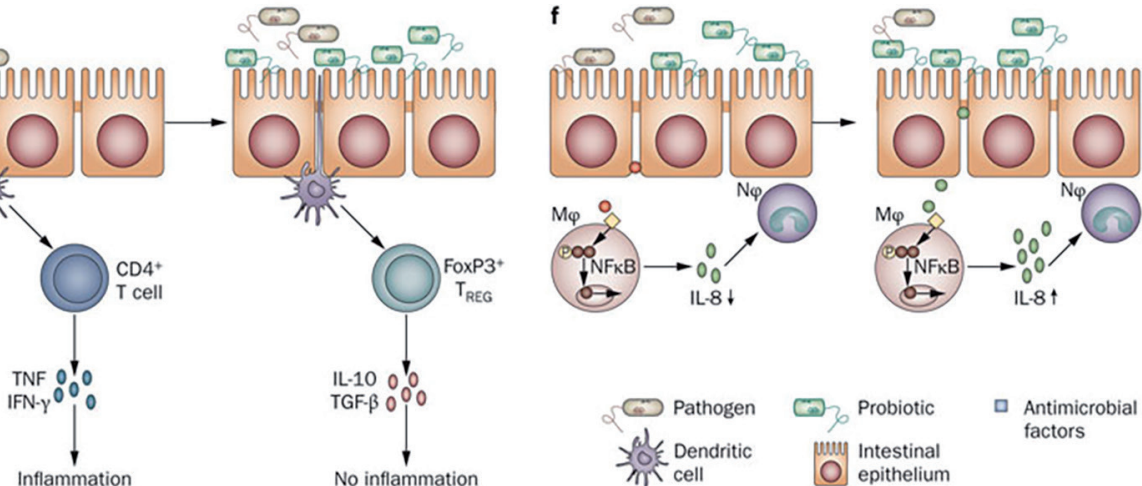

No inflammation

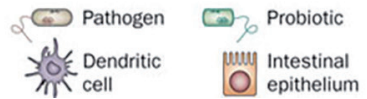

ㅁ. Antimicrobial factors

Figure 2. Potential mechanisms of action of probiotics. Probiotic organisms can provide a beneficial effect on intestinal epithelial cells in numerous ways. a: Some strains can block pathogen entry into the epithelial cell by providing a physical barrier, referred to as colonization resistance or $\mathbf{b}$ : create a mucus barrier by causing the release of mucus from goblet cells. c: Other probiotics maintain intestinal permeability by increasing the intercellular integrity of apical tight junctions, for example, by upregulating the expression of zona-occludens 1 (a tight junction protein), or by preventing tight junction protein redistribution thereby stopping the passage of molecules into the lamina propria. d: Some probiotic strains have been shown to produce antimicrobial factors. e: Still other strains stimulate the innate immune system by signaling dendritic cells, which then travel to mesenteric lymph nodes and lead to the induction of TREG cells and the production of anti-inflammatory cytokines, including IL-10 and TGF- $\beta$. f: Some probiotics (or their products) may also prevent (left-hand side) or trigger (right-hand side) an innate immune response by initiating TNF production by epithelial cells and inhibiting (or activitating) $\mathrm{NF} \kappa \mathrm{B}$ in $\mathrm{M} \varphi$ and dampening (or priming) the host immune response by influencing the production of IL- 8 and subsequent recruitment of $N \varphi$ to sites of intestinal injury. Abbreviations: M $\varphi$, macrophage; N $\varphi$, neutrophil; TREG cell, regulatory T cell. Reproduced from, Gareau M. G., P. M. Sherman \& W. A. Walker (2010) Nature Reviews Gastroenterology and Hepatology 7, 503-514.

\section{Probiotics and gut health}

\subsection{Gut microbiota}

The human gastrointestinal tract is inhabited by a complex and dynamic population of around 500-1000 of different microbial species which remain in a complex equilibrium. It 
has been estimated that bacteria account for $35-50 \%$ of the volume content of the human colon. These include Bacteroides, Lactobacillus, Clostridium, Fusobacterium, Bifidobacterium, Eubacterium, Peptococcus, Peptostreptococcus, Escherichia and Veillonella. The bacterial strains with identified beneficial properties include mainly Bifidobacterium and Lactobacillus species. The dominant microbial composition of the intestine have been shown to be stable over time during adulthood, and the microbial patterns are unique for each individual. However, there are numerous external factors that have potential to influence the microbial composition in the gut as host genetics, birth delivery mode, diet, age, antibiotic treatments and also, other microorganisms as probiotics. (Collado et al., 2009). The intestine is one of the main surfaces of contact with exogenous agents (viruses, bacteria, allergens) in the human body. It has a primary role in the host defense against external aggressions by means of the intestinal mucosa, the local immune system, and the interactions with the intestinal microbiota (resident and in transitbacteria). Gut microbiota influences human health through an impact on the gut defense barrier, immune function, nutrient utilization and potentially by direct signaling with the gastrointestinal epithelium (Collado et al., 2009). Only a limited fraction of bacterial phyla compose the major intestinal microbiota. In healthy adults, $80 \%$ of phylotypes belong to four major phylogenetic groups, which are the Clostiridium leptum, Clostridium coccoides, Bacteroides and Bifidobacteria groups. However, a large fraction of dominant phylotypes is subject specific. Also, studies have found that mucosal microbiota is stable along the distal gastrointestinal tract from ileum to rectum, but mucosa-associated microbiota is different from fecal microbiota. The difference has been estimated to be between $50-90 \%$.

The intestinal microbiota is not homogeneous. The number of bacterial cells present in the mammalian gut shows a continuum that goes from $10^{1}$ to $10^{3}$ bacteria per gram of contents in the stomach and duodenum, progressing to $10^{4}$ to $10^{7}$ bacteria per gram in the jejunum and ileum and culminating in $10^{11}$ to $10^{12}$ cells per gram in the colon (Figure 3a). Additionally, the microbial composition varies between these sites. In addition to the longitudinal heterogeneity displayed by the intestinal microbiota, there is also a great deal of latitudinal variation in the microbiota composition (Figure 3b). The intestinal epithelium is separated from the lumen by a thick and physicochemically complex mucus layer. The microbiota present in the intestinal lumen differs significantly from the microbiota attached and embedded in this mucus layer as well as the microbiota present in the immediate proximity of the epithelium. For instance, Bacteroides, Bifidobacterium, Streptococcus, members of Enterobacteriacea, Enterococcus, Clostridium, Lactobacillus, and Ruminococcus were all found in feces, whereas only Clostridium, Lactobacillus, and Enterococcus were detected in the mucus layer and epithelial crypts of the small intestine (Sekirov et al., 2010). Colonization of the human gut with microbes begins immediately at birth (Figure 3c). Upon passage through the birth canal, infants are exposed to a complex microbial population. After the initial establishment of the intestinal microbiota and during the first year of life, the microbial composition of the mammalian intestine is relatively simple and varies widely between different individuals and also with time. However, after one year of age, the intestinal microbiota of children starts to resemble that of a young adult and stabilizes (Figure 3c) (Sekirov et al., 2010). 


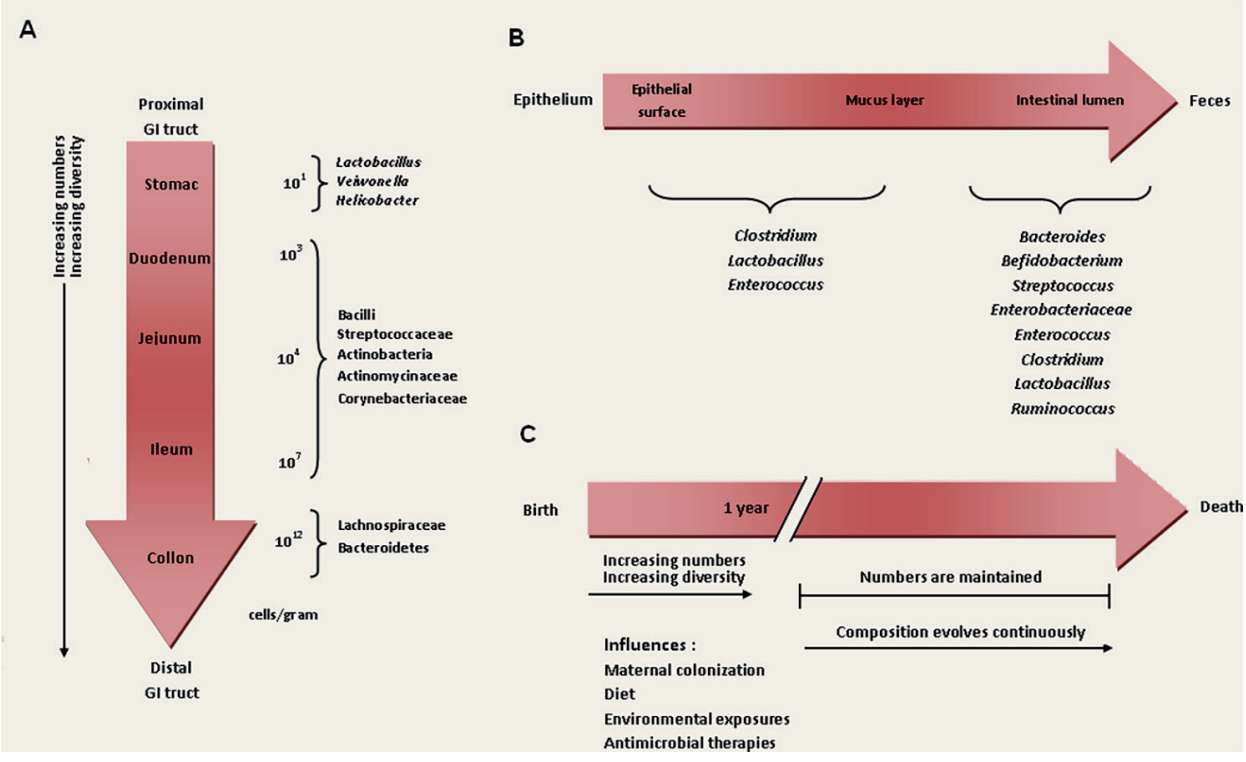

Figure 3. Spatial and temporal aspects of intestinal microbiota composition. A: variations in microbial numbers and composition across the length of the gastrointestinal tract. B: longitudinal variations in microbial composition in the intestine. C: temporal aspects of microbiota establishment and maintenance and factors influencing microbial composition (Sekirov et al., 2010).

\subsection{Survival and antagonism effects of probiotics in the gut}

The intestinal epithelium is the largest mucosal surface in the human body, provides an interface between the external environment and the host. The gut epithelium is constantly exposed to foreign microbes and antigens derived from digested foods. Thus, the gut epithelium acts as a physical barrier against microbial invaders and is equipped with various elements of the innate defense system. In the gut, two key elements govern the interplay between environmental triggers and the host: intestinal permeability and intestinal mucosal defense. Resident bacteria can interact with pathogenic microorganisms and external antigens to protect the gut using various strategies.

According to the generally accepted definition of a probiotic, the probiotic microorganism should be viable at the time of ingestion to confer a health benefit (Danisco, 2013). Although not explicitly stated, this definition implies that a probiotic should survive GI tract passage and, colonize the host epithelium. A variety of traits are believed to be relevant for surviving GI tract passage, the most important of which is tolerance both to the highly acidic conditions present in the stomach and to concentrations of bile salts found in the small intestine. These properties have consequently become important selection criteria for new probiotic functionality (Piątek et al., 2012). In addition to tolerating the harsh physical-chemical environment of the GI tract, adherence to intestinal mucosal cells would be necessary for colonization and any direct interactions between the probiotic and host cells leading to the competitive exclusion of pathogens and/or modulation of host cell responses. Moreover, As enteropathogenic 
Escherichia coli are known to bind to epithelial cells via mannose receptors, probiotic strains with similar adherence capabilities could inhibit pathogen attachment and colonization at these binding sites and thereby protect the host against infection (Marco et al., 2006).

Probiotic bacteria can antagonize pathogenic bacteria by reducing luminal $\mathrm{pH}$, inhibiting bacterial adherence and translocation, or producing antibacterial substances and defensins. One of the mechanisms by which the gut flora resists colonization by pathogenic bacteria is by the production of a physiologically restrictive environment, with respect to $\mathrm{pH}$, redox potential, and hydrogen sulfide production. Probiotic bacteria decrease the luminal $\mathrm{pH}$, as has been demonstrated in patients with ulcerative colitis (UC) following ingestion of the probiotic preparation VSL\#3. In a fatal mouse Shiga toxin-producing E. coli O157:H7 infection model, the probiotic Befidobacterium breve produced a high concentration of acetic acid, consequently lowering the luminal $\mathrm{pH}$. This $\mathrm{pH}$ reduction was associated with increased animal survival ( $\mathrm{Ng}$ et al., 2009).

Production of antimicrobial compounds, termed bacteriocins, by probiotic bacteria is also likely to contribute to their beneficial activity. Several bacteriocins produced by different species from the genus Lactobacillus have been described. The inhibitory activity of these bacteriocins varies; some inhibit taxonomically related Gram-positive bacteria, and some are active against a much wider range of Gram-positive and Gram-negative bacteria as well as yeasts and molds. For example, the probiotic L. salivarius subsp. salivarius UCC118 produces a peptide that inhibits a broad range of pathogens such as Bacillus, Staphylococcus, Enterococcus, Listeria, and Salmonella species. Lacticin 3147, a broad-spectrum bacteriocin produced by Lactococcus lactis, inhibits a range of genetically distinct Clostridium difficile isolates from healthy subjects and patients with IBD. A further example is the antimicrobial effect of Lactobacillus species on I infection of gastric mucosa, achieved by the release of bacteriocins and the ability to decrease adherence of this pathogen to epithelial cells (Gotteland et al., 2006). Probiotics can reduce the epithelial injury that follows exposure to E. coli O157:H7 and E. coli O127:H6. The pretreatment of intestinal (T84) cells with lactic acid-producing bacteria reduced the ability of pathogenic E. coli to inject virulence factors into the cells or to breach the intracellular tight junctions. Adhesion and invasion of an intestinal epithelial cell line (Intestine 407) by adherent invasive E. coli isolated from patients with Crohn's disease (CD) was substantially diminished by co- or preincubation with the probiotic strain E. coli Nissle 1917 (Wehkamp et al., 2004; Schlee et al., 2007). These findings demonstrate that probiotics prevent epithelial injury induced by attaching-effacing bacteria and contributes to an improved mucosal barrier and provide a means of limiting access of enteric pathogens (Sherman et al., 2005).

\section{Probiotics and the mucous layer}

Most mucosal surfaces are covered by a hydrated gel formed by mucins. Mucins are secreted by specialized epithelial cells, such as gastric foveolar mucous cells and intestinal goblet cells, Goblet cells are found along the entire length of the intestinal tract, as well as other mucosal surfaces. Mucins, are abundantly core glycosylated (up to $80 \% \mathrm{wt} / \mathrm{wt}$ ) and either localized to the cell membrane or secreted into the lumen to form the mucous layer (Turner, 2009). Of the 18 mucin-type glycoproteins expressed by humans, MUC2 is the predominant glycoprotein 
found in the small and large bowel mucus. The NH2- and COOH-termini are not glycosylated to the same extent, but are rich in cysteine residues that form intra- and inter-molecular disulfide bonds. These glycan groups confer proteolytic resistance and hydrophilicity to the mucins, whereas the disulfide linkages form a matrix of glycoproteins that is the backbone of the mucous layer (Ohland and MacNaughton, 2010). Although small molecules pass through the heavily glycosylated mucus layer with relative ease, bulk fluid flow is limited and thereby contributes to the development of an unstirred layer of fluid at the epithelial cell surface. As the unstirred layer is protected from convective mixing forces, the diffusion of ions and small solutes is slowed (Turner, 2009). This gel layer provides protection by shielding the epithelium from potentially harmful antigens and molecules including bacteria from directly contacting the epithelial cell layer, while acting as a lubricant for intestinal motility. Mucins can also bind the epithelial cell surface carbohydrates and form the bottom layer, which is firmly attached to the mucosa, whereas the upper layer is loosely adherent. The mucus is the first barrier that intestinal bacteria meet, and pathogens must penetrate it to reach the epithelial cells during infection (Ohland and MacNaughton, 2010).

Probiotics may promote mucus secretion as one mechanism to improve barrier function and exclusion of pathogens. In support of this concept, probiotics have been shown to increase mucin expression in vitro, contributing to barrier function and exclusion of pathogens. Several studies showed that increased mucin expression in the human intestinal cell lines Caco-2 (MUC2) and HT29 (MUC2 and 3), thus blocking pathogenic E. coli invasion and adherence. However, this protective effect was dependent on probiotic adhesion to the cell monolayers, which likely does not occur in vivo (Mack et al., 2003; Mattar et al., 2002). Conversely, another study showed that L. acidophilus A4 cell extract was sufficient to increase MUC2 expression in HT29 cells, independent of attachment (Kim et al., 2008). Additionally, intestinal trefoil factor 3 (TFF3) is coexpressed with MUC2 by colonic goblet cells and is suggested to promote wound repair (Gaudier et al., 2005 ; Kalabis et al., 2006). However, healthy rats did not display increased colonic TFF3 expression after stimulation by VSL\#3 probiotics (Caballero-Franco et al., 2007). Furthermore, mice treated with $1 \%$ dextran sodium sulfate (DSS) to induce chronic colitis did not exhibit increased TFF3 expression or wound healing when subsequently treated with VSL\#3. This observation indicates that probiotics do not enhance barrier function by up-regulation of TFF3, nor are they effective at healing established inflammation. Therefore, use of current probiotics is likely to be effective only in preventing inflammation as shown by studies in animal models (Ohland and MacNaughton, 2010).

\section{Interaction of probiotic bacteria with gut epithelium}

The composition of the commensal gut microbiota is probably influenced by the combination of food practices and other factors like the geographical localization, various levels of hygiene or various climates. The host-microbe interaction is of primary importance during neonatal period. The establishment of a normal microbiota provides the most substantial antigenic challenge to the immune system, thus helping the gut associated lymphoid tissus (GALT) maturation. The intestinal microbiota contributes to the anti-inflammatory character 
of the intestinal immune system. Several immunoregulatory mechanisms, including regulatory cells, cytokines, apoptosis among others, participate in the control of immune responses by preventing the pathological processes associated with excessive reactivity. An interesting premise for probiotic physiological action is their capacity to modulate the immune system. Consequently, many studies have focused on the effects of probiotics on diverse aspects of the immune response. Following consumption of probiotic products, the interaction of these bacteria with intestinal enterocytes initiates a host response, since intestinal cells produce various immunomodulatory molecules when stimulated by bacteria (Delcenseri et al., 2008). Furthermore, The indigenous microbiota is a natural resistance factor against potential

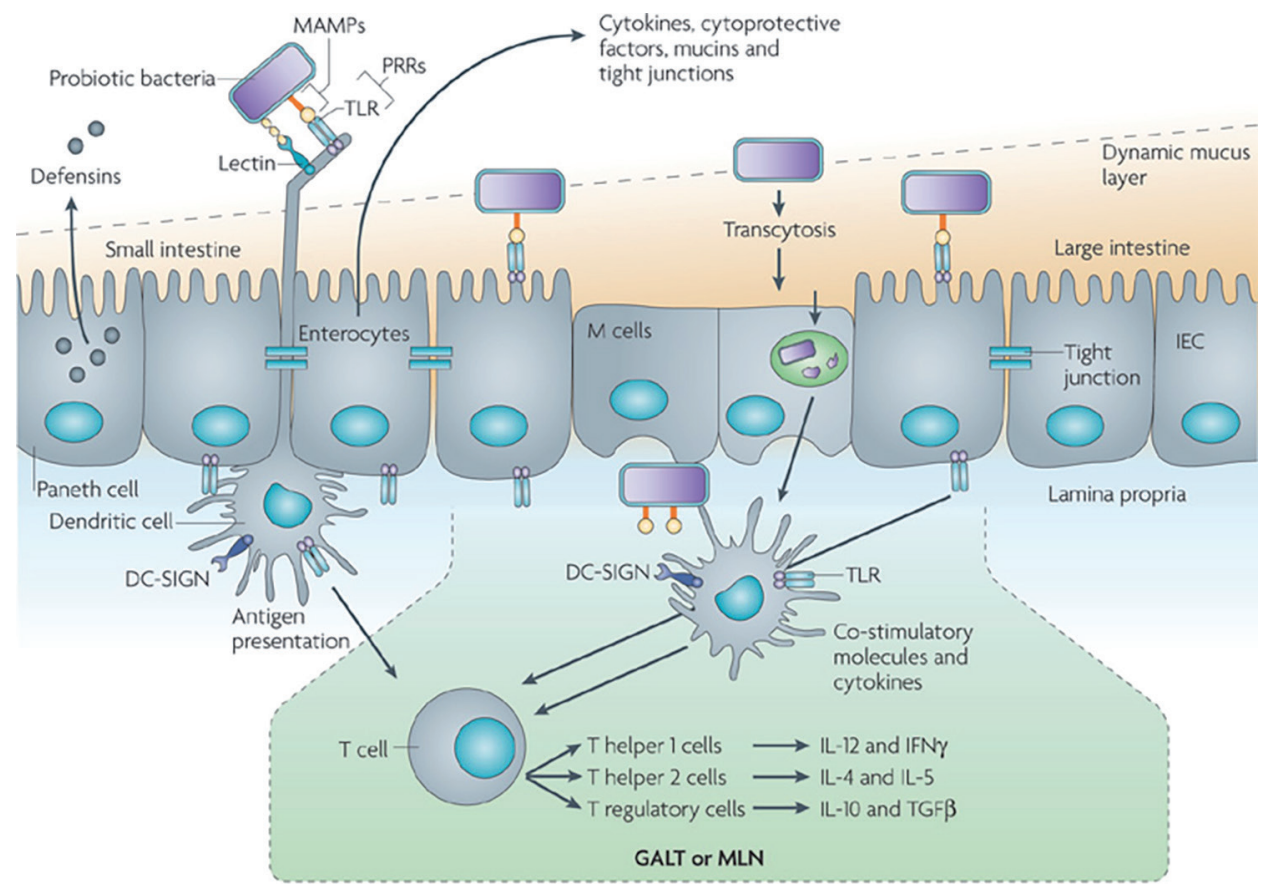

Figure 4. Interaction of probiotic bacteria with IECs and DCs from the GALT. A fraction of ingested probiotics are able to interact with intestinal epithelial cells (IECs) and dendritic cells (DCs), depending on the presence of a dynamic mucus layer. Probiotics can occasionally encounter DCs through two routes: DCs residing in the lamina propria sample luminal bacterial antigens by passing their dendrites between IECs into the gut lumen, and DCs can also interact directly with bacteria that have gained access to the dome region of the gut-associated lymphoid tissue (GALT) through specialized epithelial cells, termed microfold or $\mathrm{M}$ cells. The interaction of the host cells with microorganism-associated molecular patterns (MAMPs) that are present on the surface macromolecules of probiotic bacteria will induce a certain molecular response. The host pattern recognition receptors (PRRs) that can perceive probiotic signals include Toll-like receptors (TLRs) and the $\mathrm{C}$ type lectin DC-specific intercellular adhesion molecule 3-grabbing non-integrin (DC-SIGN). Some molecular responses of IECs depend on the subtype of cell, for example, Paneth cells produce defensins and goblet cells produce mucus. Important responses of DCs against probiotics include the production of cytokines, major histocompatibility complex molecules for antigen presentation, and co-stimulatory molecules that polarize T cells into $\mathrm{T}$ helper or CD4+CD25+ regulatory T cells in the mesenteric lymph nodes (MLNs) or subepithelial dome of the GALT. IFN $\gamma$, interferon- $\gamma$; IL, interleukin; TGFb; transforming growth factor- $\beta$. Reproduced from: S. Lebeer, J. Vanderleyden E S. C. J. De Keersmaecker (2010). Host interactions of probiotic bacterial surface molecules: comparison with commensals and pathogens. Nature Reviews Microbiology 8, 171-184. 
pathogenic microorganisms and provides colonization resistance, also known as gut barrier, by controlling the growth of opportunistic microorganisms. It has been suggested that commensal bacteria protect their host against microbial pathogens by interfering with their adhesion and toxic effects (Myllyluoma, 2007).

The tight epithelial cell barrier forms the another line of defence between the gut luminal contents and the host. Epithelial cells lining the gastrointestinal tract are able to respond to infection by initiating either nonspecific or specific host-defence response (Kagnoff and Eckmann 1997, Strober 1998). Bacterial adhesion to the host cell or recognition by the host cell is often an essential first stage in the disease process. A wide range of gastrointestinal cell surface constituents, such as several glygoconjucates, can serve as receptors for bacterial adherence (Servin and Coconnier 2003, Pretzer et al., 2005). Furthermore, epithelial cells express constitutively host pattern recognition receptors (PRRS), such as Toll-like receptors (TLR). These are a family of transmembrane receptors that recognize repetitive patterns, i.e. the pathogen-associated molecular patterns present in diverse microbes, including grampositive and gram-negative bacteria (Bäckhed and Hornef 2003, Takeda et al., 2003). TLRs are also found on innate immune cells, such as dendritic cells and macrophages (Vinderola et al., 2005). TLR4 recognizes lipopolysaccharide and gram-negative bacteria, while TLR2 recognizes a variety of microbial components, such as peptidoglycan and lipoteichoic acids, from gram-positive bacteria (Abreu 2003, Matsuguchi et al., 2003, Takeda et al., 2003). Also, several other TLRs with specific actions are known, such as TLR5, which responds to the bacterial flagella (Rhee et al., 2005), and TLR9, which is activated by bacterially derived short DNA fragments containing CpG sequences (Pedersen et al., 2005). Other known recognition receptors are nucleotide-binding oligomerization domain proteins, which recognize both grampositive and gram-negative bacteria. They are located in cell cytoplasm and are implicated in the induction of defensins. Increased epithelial barrier permeability is frequently associated with gastrointestinal disorders contributing to both disease onset and persistence (Lu and Walker 2001, Berkes 2003). The gatekeeper of the paracellular pathway is the tight junction, which is an apically located cell-cell junction between epithelial cells. The tight junction permits the passage of small molecules, such as ions, while restricting the movement of large molecules, such as antigens and microorganisms, which can cause inflammation. The integral membrane protein family, which are mainly claudins, occluding and zonula occludens 1, are implicated in the formation of the paracellular channels (Berkes et al., 2003).

\section{Origine and safety of probiotics}

An old dogma of probiotic selection has been that the probiotic strains should be of "human origin". One may argue that from evolutionary point of view, describing bacteria to be of human origin does not make much sense at all. The requirement for probiotics to be of human origin relates actually to the isolation of the strain rather than the "origin" itself. Usually, the strains claimed to be "of human origin" have been isolated from faecal samples of healthy human subjects, and have therefore been considered to be "part of normal healthy human 
gut microbiota". In reality the recovery of a strain from a faecal sample does not necessarily mean that this strain is part of the normal microbiota of this individual, since microbes passing the GI tract transiently can also be recovered from the faecal samples (Forssten et al., 2011). In practice it is impossible to know the actual origin of the probiotic strains, regardless of whether they have been isolated from faecal samples, fermented dairy products or any other source for that matter. Isolation of a strain from faeces of a healthy individual is also not a guarantee of the safety of the strain-such a sample will also always contain commensal microbes which can act as opportunistic pathogens, or even low levels of true pathogens, which are present in the individual at sub-clinical levels. Therefore, it has been recommend that instead of concentrating on the first point of isolation, the selection processes for new potential probiotic strains should mainly focus on the functional properties of the probiotic strains rather than the "origin" (Forssten et al., 2011; Ouwehand and Lahtinen 2008).

As viable, probiotic bacteria have to be consumed in large quantities, over an extended period of time, to exert beneficial effects; the issue of the safety of these microorganisms is of primary concern (Leroy et al., 2008). Until now, reports of a harmful effect of these microbes to the host are rare. However, many species of the genera Lactobacillus, Leuconostoc, Pediococcus, Enterococcus, and Bifidobacterium were isolated frequently from various types of infective lesions. According to Gasser (1994), L. rhamnosus, L. acidophilus, L. plantarum, L. casei, Lactobacillus paracasei, Lactobacillus salivarius, Lactobacillus lactis, and Leuconostoc mesenteroides are some examples of probiotic bacteria isolated from bacterial endocarditis; L. rhamnosus, L. plantarum, Leuconostoc. mesenteroides, Pediococcus acidilactici, Bifidobacterium eriksonii, and Bifidobacterium adolescentis have been isolated from bloodstream infections and many have been isolated from local infections. Although minor side effects of the use of probiotics have been reported, infections with probiotic bacteria occur and invariably only in immunocompromised patients or those with intestinal bleeding (Leroy et al., 2008).

An issue of concern regarding the use of probiotics is the presence of chromosomal, transposon, or plasmid-located antibiotic resistance genes amongst the probiotic microorganisms. At this moment, insufficient information is available on situations in which these genetic elements could be mobilised, and it is not known if situations could arise where this would become a clinical problem (Leroy et al., 2008). When dealing with the selection of probiotic strains, the FAO/WHO Consultancy recommends that probiotic microorganisms should not harbor transmissible drug resistance genes encoding resistance to clinically used drugs (FAO/WHO, 2002). For the assessment of the safety of probiotic microorganisms and products, FAO/WHO has formulated guidelines, recommending that probiotic strains should be evaluated for a number of parameters, including antibiotic susceptibility patterns, toxin production, metabolic and haemolytic activities, and infectivity in immunocompromised animals (FAO/WHO, 2002). In vitro safety screenings of probiotics may include, among others, antibiotic resistance assays, screenings for virulence factors, resistance to host defence mechanisms and induction of haemolysis. Several different animal models have been utilized in the safety assessment of probiotics. These include models of immunodeficiency, endocarditis, colitis and liver injury. In some cases even acute toxicity of probiotics has been assessed. Last but not least, also clinical intervention trials have yielded evidence on the safety of probiotics for human consumption (Forssten et al., 2011). 


\section{Conclusion}

The individual diversity of the intestinal microflora underscores the difficulty of identifying the entire human microbiota and poses barriers to this field of research. In addition, it is apparent that the actions of probiotics are species and strain specific. It is also apparent that even a single strain of probiotic may exert its actions via multiple, concomitant pathways. Probiotics have long been used as an alternative to traditional medicine with the goal of maintaining enteric homeostasis and preventing disease. However, the actual efficacy of this treatment in still debated. Clinical trials have shown that probiotic treatment can reduce the risk of some diseases, especially antibiotic-associated diarrhea, but conclusive evidence is impeded owing to the wide range of doses and strains of bacteria used. The mechanism of action is also an area of interest (Ohland and MacNaughton, 2010). Many studies, as discussed above, have shown that probiotics increase barrier function in terms of increased mucus, antimicrobial peptides, and sIgA production, competitive adherence for pathogens, and increased TJ integrity of epithelial cells. Current investigation into the mechanism of action of specific probiotics has focused on probiotic-induced changes in the innate immune functions involvingTLRs and its downstream systems Like NF- $\kappa$ B, and other pathways (Yoon and Sun, 2011). Although the immunomodulatory effects of probiotics have been demonstrated in experimental animal models of allergy, autoimmunity, and IBD, information from clinical trials in humans is scarce. Furthermore, some studies suggest that probiotics could induce detrimental effects. Therefore, more research, especially in the form of well-designed clinical trials, is needed to evaluate the efficacy and safety of probiotics (Ezendam and Van Loveren, 2008). With evolving knowledge, efective probiotic therapy will be possible in the future.

\section{Author details}

Daoud Harzallah and Hani Belhadj

Laboratory of Applied Microbiology, Faculty of Natural and Life Sciences, University Ferhat Abbas, Sétif, Algeria

\section{References}

[1] Abreu M.T. (2003). Immunologic regulation of toll-like receptors in gut epithelium. Curr. Opin. Gastroenterol; 19:559-64.

[2] Bäckhed F, and M. Hornef (2003). Toll-like receptor 4-mediated signaling by epithelial surfaces: necessity or threat? Microbes Infect.; 5:951-9.

[3] Belhadj, H., Harzallah, D., Khennouf, S., Dahamna, S., Bouharati, S., and Baghiani, A. (2010). Isolation, identification and antimicrobial activity of lactic acid bacteria from Algerian honeybee collected pollen. Acta Hort. (ISHS) 854: 51 - 58 
[4] Berkes J, V.K. Viswanathan, S.D. Savkovic, G. Hecht (2003). Intestinal epithelial responses to enteric pathogens: effects on the tight junction barrier, ion transport, and inflammation. Gut;52:439-51.

[5] Caballero-Franco C., K. Keller, C. De Simone, K. Chadee (2007). The VSL\#3 probiotic formula induces mucin gene expression and secretion in colonic epithelial cells. Am J Physiol Gastrointest Liver Physiol 292: G315-G322.

[6] Chow J., (2002). Probiotics and prebiotics: A brief overview, J. Ren. Nutr. 12 76-86.

[7] Collado M. C., E. Isolauri, S. Salminen and Y. Sanz (2009). The Impact of Probiotic on Gut Health. Current Drug Metabolism, 10, 68-78.

[8] Desai A. (2008). Strain Identification, Viability And Probiotics Properties Of Lactobacillus Casei. PhD Thesis Victoria University, Victoria, Australia 228 pp.

[9] Danisco. (2013). Lactobacillus acidophilus La-14. Technical Memorandum. TM48-le. Available from: http://ss1.spletnik.si/4_4/000/000/19f/152/La-14\%20TM.pdf

[10] Delcenserie V., D. Martel, M. Lamoureux, J. Amiot, Y. Boutin and D. Roy (2008). Immunomodulatory effects of probiotics in the intestinal tract. Curr Issues Mol Biol., 10(1-2): 37-54. PMID: 18525105.

[11] Ezendam J., and H. Van Loveren (2008). Probiotics: Immunomodulation and Evaluation of Safety and Efficacy. Nutrition Reviews, 64, (1) : 1-14.

[12] FAO/WHO. (2002). Guidelines for the evaluation of probiotics in food - Joint Food and Agricultural Organization of the United Nations and World Health Organization Working Group Meeting Report, London Ontario, Canada.

[13] Forssten S. D., S. J. Lahtinen A. C. Ouwehand, and J. J. Malago et al. (eds.), (2011) Chapter 2 :The Intestinal Microbiota and Probiotics 24 pp. In : Probiotic Bacteria and Enteric Infections. Springer Science Business Media B.V.

[14] Gareau M. G., P. M. Sherman and W. A.Walker (2010). Probiotics and the Gut Microbiota in Intestinal Health and Disease. Nature Reviews Gastroenterology and Hepatology 7, 503-514.

[15] Gasser F. (1994). Safety of lactic acid bacteria and their occurrence in human clinical infections. Bull Inst Pasteur; 92:45-67.

[16] Gaudier E., C. Michel, JP. Segain, C. Cherbut, and C. Hoebler (2005). The VSL\# 3 probiotic mixture odifies microflora but does not heal chronic dextran- sodium sulfate-induced colitis or reinforce the mucus barrier in mice. J Nutr. 135: 2753-2761.

[17] Gotteland M., O. Brunser, S. Cruchet (2006). Systematic review: are probiotics useful in controlling gastric colonization by Helicobacter pylori? Aliment. Pharmacol. Ther.23:1077-1086.

[18] Holzapfel, W.H., P. Haberer, R. Geisen, J. Björkroth, U. Schillinger (2001) Taxonomy and important features of probiotic microorganisms in food nutrition. Am. J. Clin. Nutr. 73, 365S-373S. 
[19] Kagnoff M.F., and L. Eckmann (1997) Epithelial cells as sensors for microbial infection. J Clin Invest;100:6-10.

[20] Kalabis J, I. Rosenberg, and DK. Podolsky (2006). Vangl1 protein acts as a downstream effector of intestinal trefoil factor (ITF)/TFF3 signaling and regulates wound healing of intestinal epithelium. J Biol Chem 281:6434-6441.

[21] Kim Y, SH. Kim, KY. Whang, YJ. Kim, and S. Oh (2008). Inhibition of Escherichia coli O157:H7 attachment by interactions between lactic acid bacteria and intestinal epithelial cells. J Microbiol Biotechnol 18: 1278-1285.

[22] Kollath, W. (1953) Ernährung und Zahnsystem. Deutsche Zahnärztliche Zeitschrift 8: 7-16.

[23] Lebeer S., J. Vanderleyden and S. C. J. De Keersmaecker (2010). Host interactions of probiotic bacterial surface molecules: comparison with commensals and pathogens. Nature Reviews Microbiology 8, 171-184.

[24] Leroy, F. and De Vuyst, L. (2004). Functional lactic acid bacteria starter cultures for the food fermentation industry. Trends in Food Science and Technology 15, 67-78.

[25] Leroy F., G. Falony, and L. de Vuyst (2008) Latest Developments in Probiotics. In Toldrà F. (ed.), Meat Biotechnology, Springer Science+Business Media, LLC. 217-229 pp.

[26] Lilly D.M, and R.H. Stillwell (1965) Probiotics: growth promoting factors produced by microorganisms. Science. 147: 747-8.

[27] Lu L, and W.A. Walker (2001). Pathologic and physiologic interactions of bacteria with the gastrointestinal epithelium. Am. J. Clin. Nutr.;73:1124S-30S.

[28] Mack DR, S. Ahrne, L. Hyde, S. Wei, and MA. Hollingsworth (2003). Extracellular MUC3 mucin secretion follows adherence of Lactobacillus strains to intestinal epithelial cells in vitro. Gut 52: 827-833.

[29] Marco M. L, S. Pavan and M. Kleerebezem (2006). Towards understanding molecular modes of probiotic action . Curr Opin Biotechnol. 17:204-210.

[30] Mattar AF., DH. Teitelbaum, RA. Drongowski, F. Yongyi, CM. Harmon, and AG. Coran (2002). Probiotics up-regulate MUC-2 mucin gene expression in a Caco-2 cell-culture model. Pediatr Surg. Int. 18: 586-590.

[31] Matsuguchi T, A. Takagi, T. Matsuzaki, M. Nagaoka, K. Ishikawa, T. Yokokura, and Y. Yoshikai (2003). Lipoteichoic acids from Lactobacillus strains elicit strong tumor necrosis factor alpha-inducing activities in macrophages through Toll-like receptor 2. Clin Diagn Lab Immunol; 10:259-66.

[32] Mercenier A., S. Pavan and B. Pot (2003) Probiotics as Biotherapeutic Agents: Present Knowledge and Future Prospects. Current Pharmaceutical Design, 8 : 99-110.

[33] Metchnikoff, E. (1908). The Prolongation Of Life, New York, G.P. Putnam's Sons, 376 p.

[34] Morelli, L. (2007). In vitro assessment of probiotic bacteria: From survival to functionality. International Dairy Journal, 17, 1278-1283. 
[35] Myllyluoma E. (2007). The Role Of Probiotics In Helicobacter pylori Infection. Dessertation, 86p. Nstitute Of Biomedicine Pharmacology University Of Helsinki, Helsinki, Finland.

[36] Ng S.C., A.L. Hart, M.A. Kamm, A.J. Stagg, and S.C. Knight (2009) Mechanisms of Action of Probiotics: Recent Advances Inflamm Bowel Dis.15:300 -310.

[37] Ohland C. L. and W. K. MacNaughton (2010). Probiotic bacteria and intestinal epithelial barrier function.Am.J. Physiol. Gastrointest. Liver Physiol. 298: G807-G819.

[38] Osmanagaoglu O., and Y. Beyatli (1999) The Use of Bacteriocins Produced by Lactic Acid Bacteria in Food Biopreservation. Türk. Mikrobiyol. Cem. Derg. 32: 295-306.

[39] Ouwehand A.C., Lahtinen S.J. (2008). The (non-) sense of human origin of probiotics. NutraCos, 7:8-10.

[40] Parada J. L., C. R.Caron, A. B. P. Medeiros and C. R. Soccol (2007) Bacteriocins from Lactic Acid Bacteria: Purification, Properties and use as Biopreservatives. Brazilian Archives of Biology And Technology. Vol.50 (3):521-542.

[41] Pedersen G, L. Andresen, M.W. Matthiessen, J. Rask-Madsen, J. Brynskov (2005). Expression of Toll-like receptor 9 and response to bacterial CpG oligodeoxynucleotides in human intestinal epithelium. Clin Exp Immunol;141:298-306.

[42] Piątek J., M. Gibas-Dorna, A. Olejnik, H. Krauss, K. Wierzbicki, W. Żukiewicz-Sobczak, M. Głowacki (2012). The viability and intestinal epithelial cell adhesion of probiotic strain combination--in vitro study. Ann Agric Environ Med.; 19(1):99-102. PMID: 22462453.

[43] Pretzer G, J. Snel, D. Molenaar, A. Wiersma, P.A. Bron, J. Lambert, WM de Vos, R. Ven Der Meer, M.A. Smits, and M. Kleerebezem (2005). Biodiversity-based identification and functional characterization of the mannose-specific adhesin of Lactobacillus plantarum. J Bacteriol. 187:6128-36.

[44] Rastall R. A., R. Fuller, H. R. Gaskins, G. Gibson (2000). Colonic functional foods. In: Gibson G. and Williams CM. (eds.) Functional Foods: Concept to Product. Woodhead Publishing Limited, Cambridge; pp. 72-95.

[45] Rhee SH, E. Im, M. Riegler, E. Kokkotou, M. O’Brien, C. Pothoulakis. (2005). Pathophysiological role of Toll-like receptor 5 engagement by bacterial flagellin in colonic inflammation. Proc. Natl. Acad .Sci.; 102:13610-5.

[46] Sanders M. E. (2008). Probiotics: Definition, Sources, Selection, and Uses. Clinical Infectious Diseases. 46:S58-61.

[47] Shanahan F. (2003) Probiotics: a perspective on problems and pitfalls, Scand. J. Gastroenterol. 38 (S) :34- 36.

[48] Schlee M., J. Wehkamp, A. Altenhoefer, et al. (2007). Induction of human beta-defensin 2 by the probiotic Escherichia coli Nissle 1917 is mediated through flagellin. Infect Immun.75:2399-2407. 
[49] Schrezenmeir J., and M. De Vrese (2001) probiotics, prebiotics, and symbiotics approaching a definition. Am. J. Clin. Nutr. 73:361S-364S.

[50] Sherman PM, K.C. Johnson-Henry, H.P. Yeung, et al. (2005) Probiotics reduce enterohemorrhagic Escherichia coli O157:H7- and enteropathogenic E. coli O127:H6- induced changes in polarized T84 epithelial cell monolayers by reducing bacterial adhesion and cytoskeletal rearrangements. Infect Immun.73:5183-5188.

[51] Sekirov I., S. L. Russell, L. C. M. Antunes, And B. B. Finlay (2010). Gut Microbiota in Health and Disease. Physiol Rev 90: 859-904.

[52] Servin A.L., M.H. Coconnier (2003). Adhesion of probiotic strains to the intestinal mucosa and interaction with pathogens. Best Pract. Res. Clin. Gastroenterol;17:741-54.

[53] Soccol C. R., L. P. de Souza Vandenberghe, M. R. Spier, A. B. Pedroni Medeiros, C. T. Yamaguishi, J. De D. Lindner, A. Pandey and V. Thomaz-Soccol1(2010). The Potential of Probiotics: A Review. Food Technol. Biotechnol. 48 (4): 413-434.

[54] Strober W. (1998). Interactions between epithelial cells and immune cells in the intestine. Ann N.Y Acad. Sci.; 859:37-45.

[55] Takeda K, T. Kaisho, and S. Akira (2003). Toll-like receptors. Annu. Rev. Immunol.; 21:335-76.

[56] Tuomola, E., R. Crittenden, M. Playne, E. Isolauri, and S. Salminen. (2001). Quality assurance criteria for probiotic bacteria. Am J Clin Nutr.; 73:393S -398S.

[57] Turner J. R. (2009). Intestinal mucosal barrier function in health and disease. Nature Reviews Immunology. 9: 799-809.

[58] Vasiljevic and N.P. Shah, (2008) Review: probiotics - from Metchnikoff to bioactives, International Dairy Journal 18, pp. 714-728.

[59] Vinderola G, C. Matar, and G. Perdigon. (2005). Role of intestinal epithelial cells in immune effects mediated by gram-positive probiotic bacteria: involvement of toll-like receptors. Clin. Diagn. Lab Immunol; 12:1075-84.

[60] Vergin F. (1954) Anti- and probiotika. Hippokrates 25: 16-19.

[61] Yoon S. S., and J. Sun (2011). Probiotics, Nuclear Receptor Signaling, and AntiInflammatory Pathways. Gastroenterology Research and Practice Volume 111: 1-16.

[62] Wehkamp J., J. Harder, K. Wehkamp, et al. (2004). NF-kappaB- and AP-1-mediated induction of human beta defensin-2 in intestinal epithelial cells by Escherichia coli Nissle 1917: a novel effect of a probiotic bacterium. Infect Immun.72:5750-5758. 